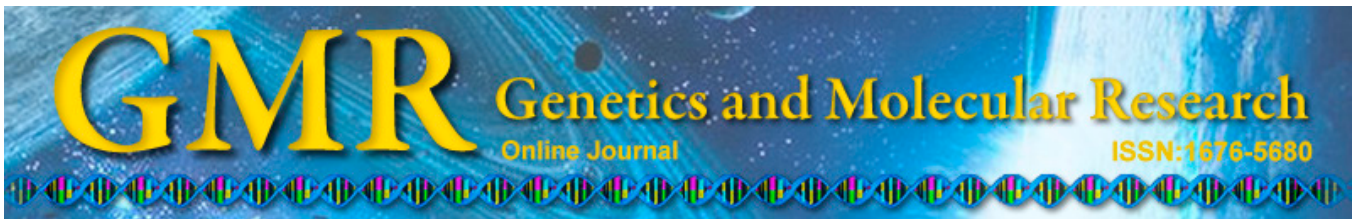

\title{
Ultrasonic imaging of gunshot wounds in pig limb
}

\author{
Q. Li, D. Deng, J. Tao, X. Wu, F. Yi, G. Wang and F. Yang \\ Department of Ultrasonography, \\ PLA Chengdu Military Area Command General Hospital, Chengdu, China \\ Corresponding author: D. Deng \\ E-mail: dandengen@163.com
}

Genet. Mol. Res. 14 (2): 4291-4302 (2015)

Received March 17, 2014

Accepted September 24, 2014

Published April 30, 2015

DOI http://dx.doi.org/10.4238/2015.April.30.1

\begin{abstract}
We investigated wound tract extension of traumatic gunshot wounds in limb soft tissues as well as wound tract sonographic features and change-patterns when the limb position was changed. The experimental animals included 8 healthy crossbred pigs in the Chengdu plain region. Chinese Type 53 Carbine was used to establish the gunshot wound model of porcine soft tissues. Gunshot-injured zones in the soft tissues were dynamically observed at different time points using ultrasonic technology. Pathological examinations were performed for the corresponding regions for comparison and analysis. The internal echo of the wound tract was a pipe-like echo that changed over time. The wound tract extension changed with postural changes. The gas echo extended along the inside of the wound track, surrounding the fascia to further tissues. Ultrasonic imaging of gunshot wounds in pig soft tissues shows specific characteristics. The application of ultrasound technology may provide important imaging protection for gunshot wound debridement and postoperative unobstructed drainage, helping to improve the judgment and treatment of limb gunshot injuries.
\end{abstract}

Key words: Experimental study; Gunshot wound; Pig limb; Ultrasonography; Wound tract 


\section{INTRODUCTION}

Limb gunshot wounds account for 40-96\% of total gunshot wounds (Stanec et al., 1993; Coupland, 1994; Truax et al., 1997). In modern society, because of social factors such as firearms smuggling and homemade bullets, the incidence of short-distance gunshot wounds has increased. Various inspection methods can be used to examine gunshot wound injury (Schwartz, 1995; Parker et al., 1997; Kim et al., 2002; Sadjadi et al., 2009; Tartaglione et al., 2012); however, imaging applications would be more suitable for clinical treatment. Under resource-limited field conditions, surgeons must evaluate injuries quickly and accurately. The large equipment required for certain methods such as computer tomography and magnetic resonance imaging is difficult to move, produces strong radiation, has complicated operation procedures, and cannot be used for on-spot rescue. In addition, if the patient's location is too far away from the rescue hospital to obtain timely treatment, changes in the patient's position during transportation may complicate the wound tract. Therefore, a method of non-invasive examination that is safe, relatively easy, and can be performed under field conditions to quickly and accurately provide injury information and real-time monitoring of wound tract changes for the debridement would be more suitable for practical clinical practical.

As modern ultrasound technology continues to improve, the application of ultrasonography for trauma evaluation has also been further developed (Tayal et al., 2004). However, ultrasound imaging studies examining the extension characteristics and change patterns of gunshot wounds in soft tissues are limited, and have only been used for relatively simple wound track imaging.

We hypothesized that because of the advantages of modern ultrasound equipment, such as high resolution, ease of transport, convenience for on-site first aid, and real-time dynamic monitoring, this technology could be used to examine gunshot wounds in the soft tissues and that sonographic characteristics and change patterns after the body position changed could be further understood. We examined whether this method could be used to facilitate the clinical treatment of limb gunshot wounds. In this study, we established a gunshot wound model of the porcine lower limb, performed routine ultrasound examinations of the injured pigs, dynamically observed the imaging characteristics of the gunshot wounds, and then compared and analyzed the pathological results of the corresponding regions.

\section{MATERIAL AND METHODS}

\section{Materials}

All experiments strictly followed the Laboratory Animal Management Ordinance of American Academy. We obtained permission from the Animal Management Committee of the hospital and referred to the "Swedish model" (the experimental model used dogs or pigs as the experimental animals, and the injury site was the lower extremity). The animals used were 8 healthy crossbred pigs from the Chengdu plain region, male and female, weighing 30 $\pm 2.3 \mathrm{~kg}$. The firearm used to induce the wound was a Chinese Type 53 Carbine (Chongqing Changjiang Machinery Factory, Chongqing, China). The standard steel ball weighed $0.15 \mathrm{~g}$, the projectile velocity was measured to be $400 \mathrm{~m} / \mathrm{s}$ at the shooting moment using an XL-1000 type network target speedometer (Xi' an Oriental Machinery Factory, Xi'an, China), and the energy absorption was $12.0 \mathrm{~J}$. 


\section{Establishment of the gunshot wound model}

Experimental animals were fasted for $6 \mathrm{~h}$; after anesthetization with ketamine hydrochloride $(10-15 \mathrm{mg} / \mathrm{kg}$ ) and $3 \%$ sodium pentobarbital $(30 \mathrm{mg} / \mathrm{kg})$, the animals were placed in the supine position and fixed on the firing stage, with one side hindlimb suspended. We targeted the thick muscular tissue of the left hind femur, and shot from the inner side of the thigh to the outside; all shootings were executed by professional shooters at a shooting distance of 2 $\mathrm{m}$. Before shooting, ultrasound was used to help identify and mark the shooting point in order to avoid direct injuries to the femur and large thigh vessels.

\section{Ultrasound examination}

For ultrasound examination, we used an HI Preirus (Hitachi, Tokyo, Japan) with a probe frequency of 7.0-13.0 MHz. All ultrasound image data were stored on the hard disk of the instrument. After successfully preparing the models, the animals were maintained in the injury posture (vertical position), and the injured site of the injured limb and the corresponding region of the contralateral limb were subjected to ultrasound scanning to identify and analyze the sonographic features of the injured region. Next, the injured position was changed to cause the injured limb to enter the flexion state (Figure 1), and the same method was used to perform ultrasound examination on the above sites. Observation time points included immediately after injury, and at $6,24,48,72$, and $168 \mathrm{~h}$.

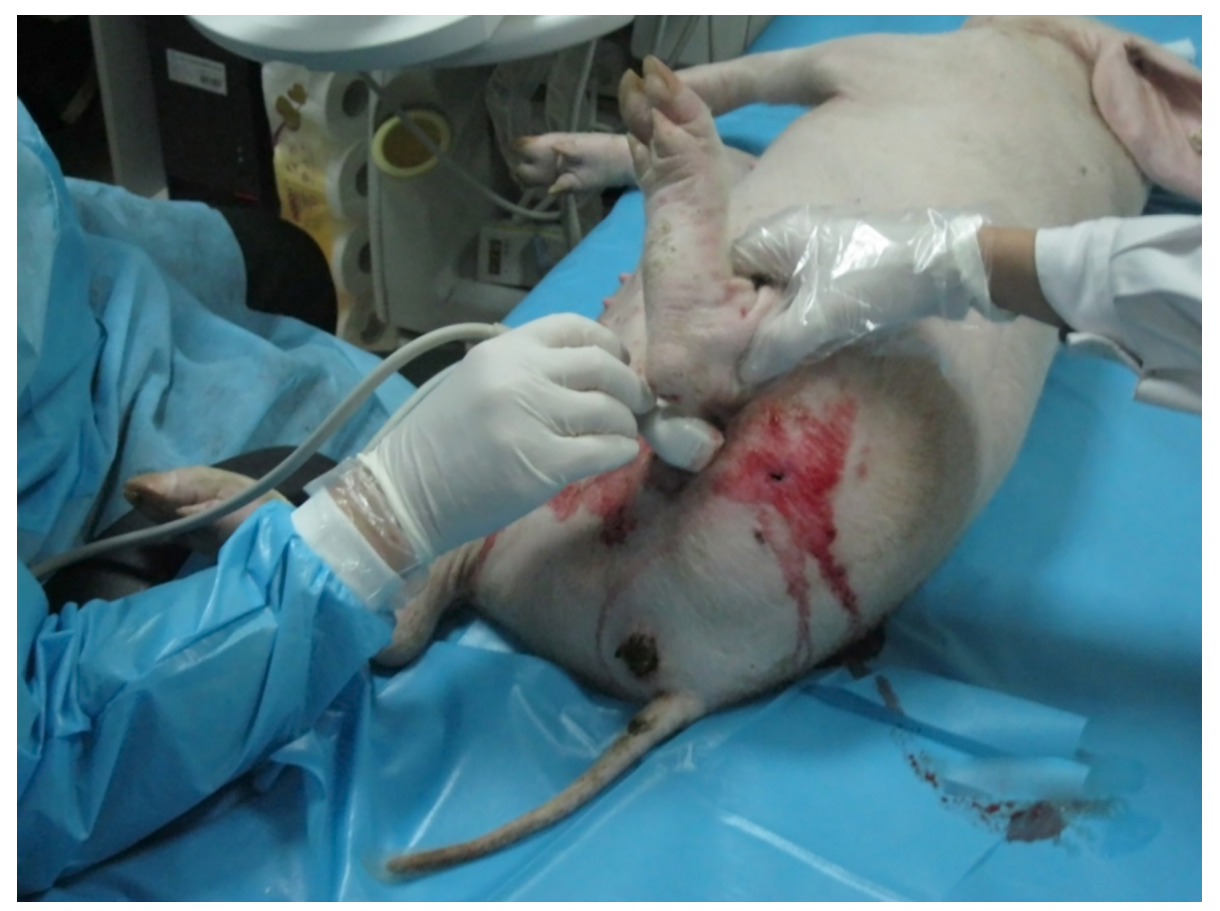

Figure 1. The posture of the pig injured limb was changed, the injured limb was in the buckling status, then the ultrasound examination was performed to observe whether the wound tract existed the changes. 


\section{Pathological examination}

After the experiment, the animals were sacrificed and dissected, the wound tract was removed for the observation of the longitudinal and cross-section characteristics, and histopathological examination was performed.

\section{RESULTS}

\section{Animal injuries}

All animal models survived and succeeded with the wound as the penetrating trauma, and the ultrasonography confirmed that there was no injury in the femur or important vessels.

\section{Ultrasonography}

The extension of the wound tract had significant sonographic features: the extension was slightly tortuous, continuous, pipe-shaped, and had a variable diameter and irregular edge (Figure 2); after changing the animal's position, the continuity of the tubular echo was interrupted immediately, and several fragments were produced which were mutually unconnected and located at some distance from each other (Figure 3), and the corresponding muscle parts of the contralateral limb showed clear movement. After the injury posture was recovered, the wound tract resumed to a continuous tubular echo.

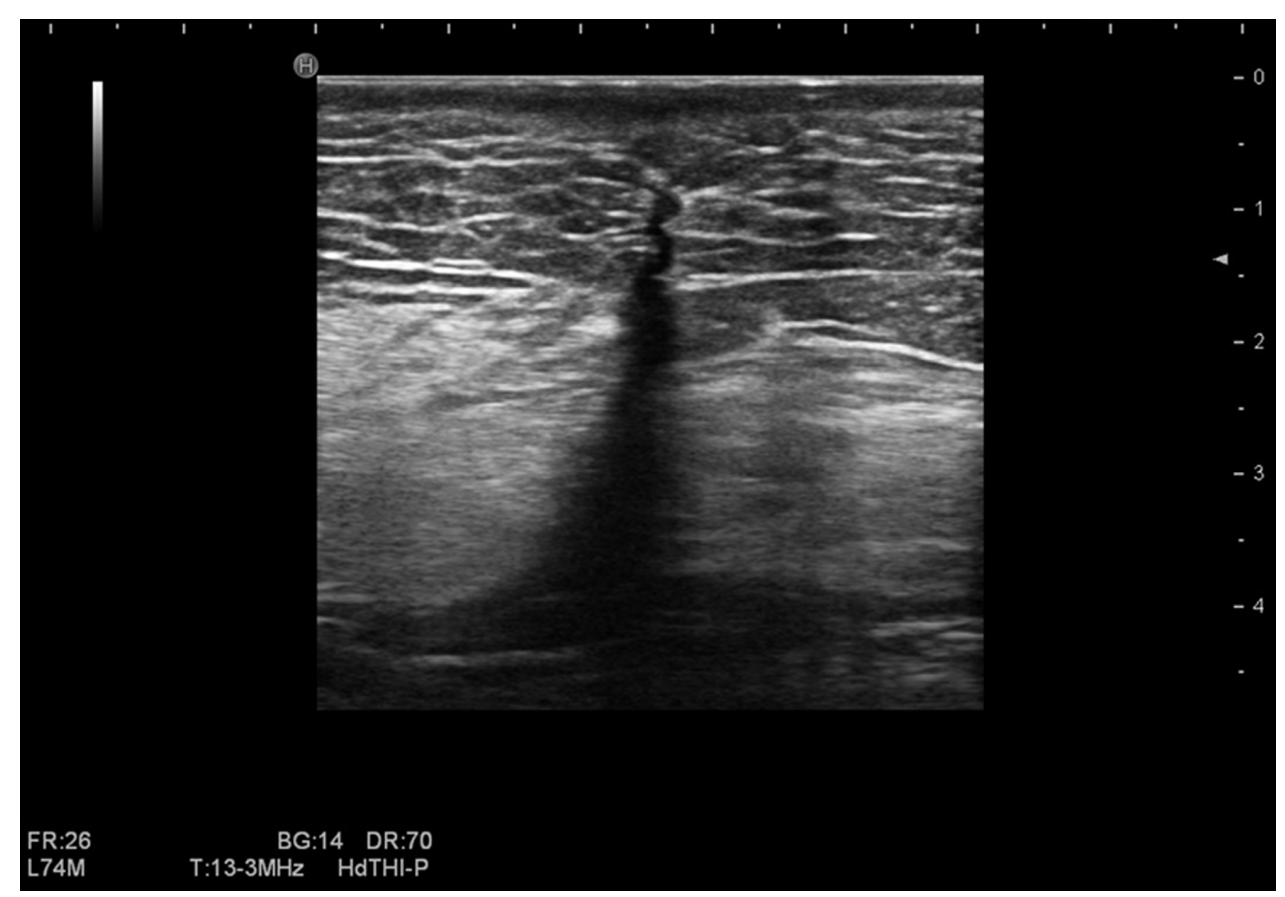

Figure 2. The wound tract was little tortuous, continuous pipe-shaped, with various diameter and irregular edge. 


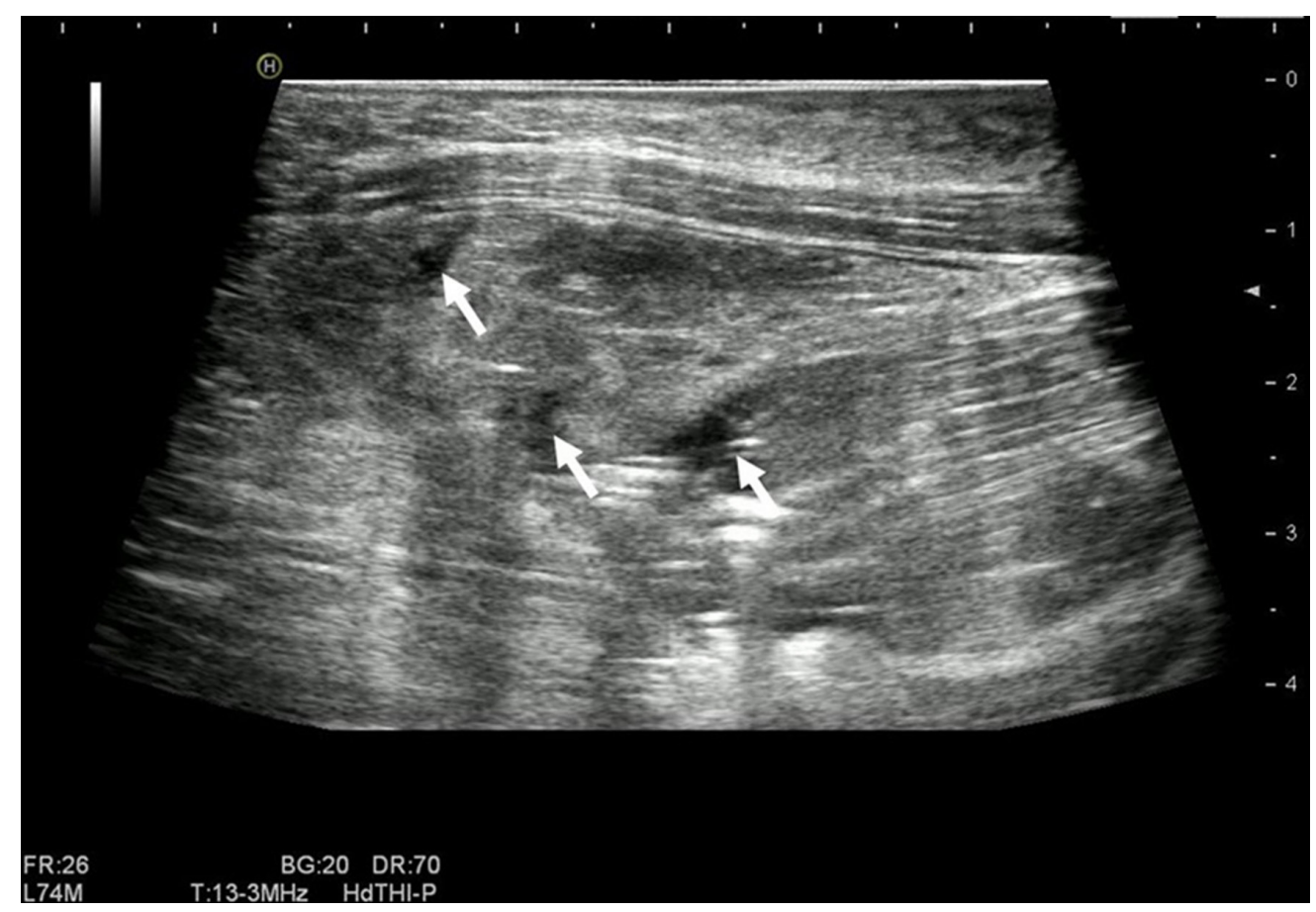

Figure 3. After the position changed, the continuity of the tubular echo was interrupted, and several fragments were produced.

\section{Ultrasonography}

Anatomical structures of the injured limb (skin, subcutaneous tissue, fascia, muscle) could be clearly distinguished. The wound tract showed the following ultrasound imaging characteristics at each time point. 1) Immediately after the injury, the main part of the wound tract appeared to have an irregular, band-shaped strong gas echo between the inlet and the outlet, some regions appeared as liquid dark areas, the peritissue spaces of the wound tract showed a scattered distribution of strong gas echo, which also existed in areas far away from the wound tract (Figure 4). At approximately $6 \mathrm{~h}$ after the formation of the wound tract, the strong gas echo in the wound tract began to decrease, while the range of the liquid dark area gradually increased. When we used a probe to place pressure on the liquid dark area, the area could be compressed, and in some sections, the strong gas echo could move among the liquid dark areas with the application of pressure. Twenty-four hours after injury, the liquid dark area in the wound tract significantly reduced, while the middle and low echo began to increase. 2) After $72 \mathrm{~h}$, the wound tract mainly appeared to as irregular hypoechogenicity, while strongest echo disappeared. The extension of the wound tract remained clear, showing a large difference from the echo of the surrounding muscle tissues, while the strong gas echo in the distant muscle gaps essentially disappeared. 3) The echo of the muscle tissue at the edge of the wound tract exhibited texture disorder, showing thin strips or lump non-echo or uneven-echo area, while the texture of the muscle tissue $0.50-0.55 \mathrm{~cm}$ away from the edge of the wound tract exhibited no significant change. 4) After the injection of saline into the wound tract, the wound 
tract and fascia adjacent to the wound tract were opened (Figure 5), and the strong gas echo in the surrounding tissues were observed to be interconnected with the wound tract.

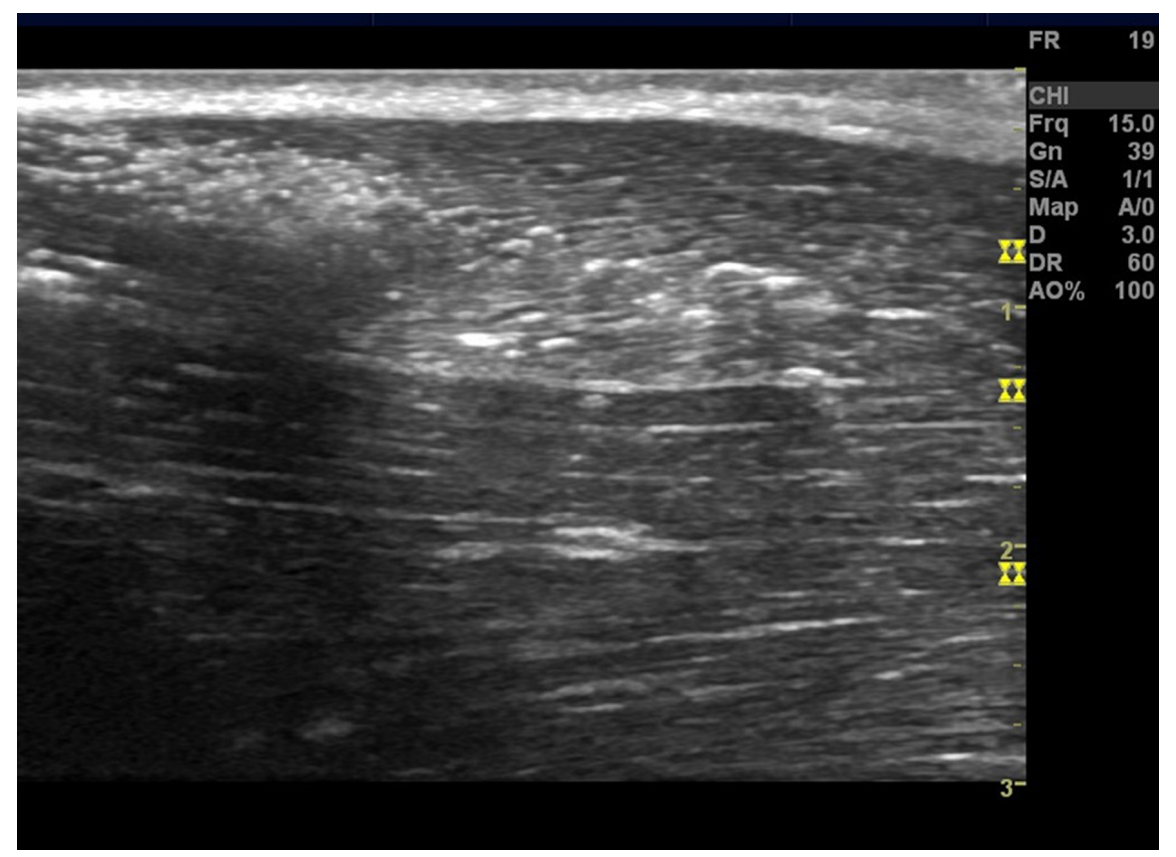

Figure 4. Strong gas echo could be seen in the distant tissues and tissue-gaps.

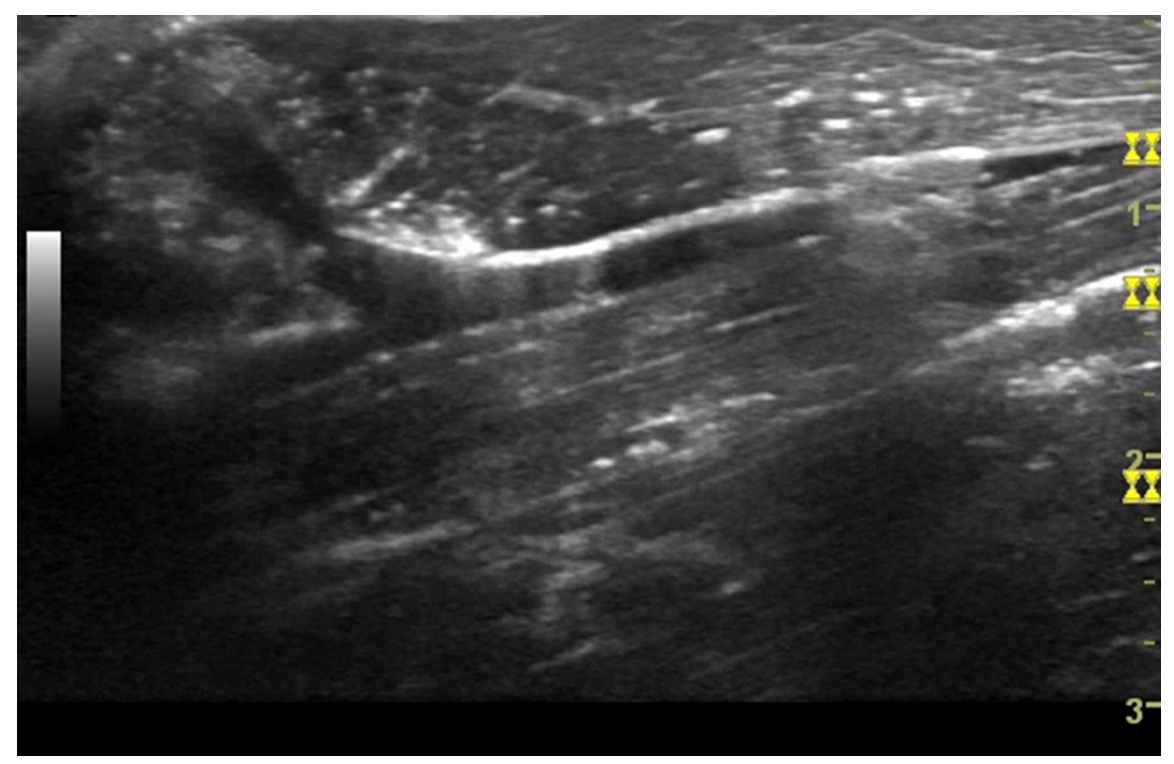

Figure 5. After the injection of saline into the wound tract, the wound tract and fascia adjacent to the wound tract were opened. 


\section{General pathological examination}

The general longitudinal section of the wound tract was approximately $0.4-1.0 \mathrm{~cm}$ wide, the inlet diameter was slightly smaller, with an inner diameter of approximately $0.4-0.5$ $\mathrm{cm}$. The outlet diameter was slightly larger, with an inner diameter of approximately $0.9-1.0$ $\mathrm{cm}$, and a slightly enlarged middle region. The edge of the wound tract inlet was neat, while the edge of outlet was irregular, mostly jagged, stellate, or showing irregular skin-tearing. In the general cross-section, a blood clot could be seen inside the wound tract, surrounded by dark brown irregular ring-shaped necrotic tissues, with a width of approximately $0.3-0.4 \mathrm{~cm}$. The outer layer of the necrotic tissues was a dark-red hyperemia band with a width of 0.3-0.6 $\mathrm{cm}$. The outer layer of the hyperemia band exhibited no obvious abnormalities with the naked eye. The scattered and various-size hematoma echo could be seen in the surrounding tissues of the wound tract.

\section{Tissue pathology after opening of the wound tract}

The pathology of the muscle tissues adjacent to the wound tract showed extensive fragmentation (Figure 6); the bleeding appeared among the muscle bundles (Figure 7) as dark brown areas around the wound tract of the general specimen. Outside this area, the muscle cells were cloudy, swelling, and loose, and the muscle cells of the partial region showed wavy changes (Figure 8). The control group showed normal pathological muscle tissues.

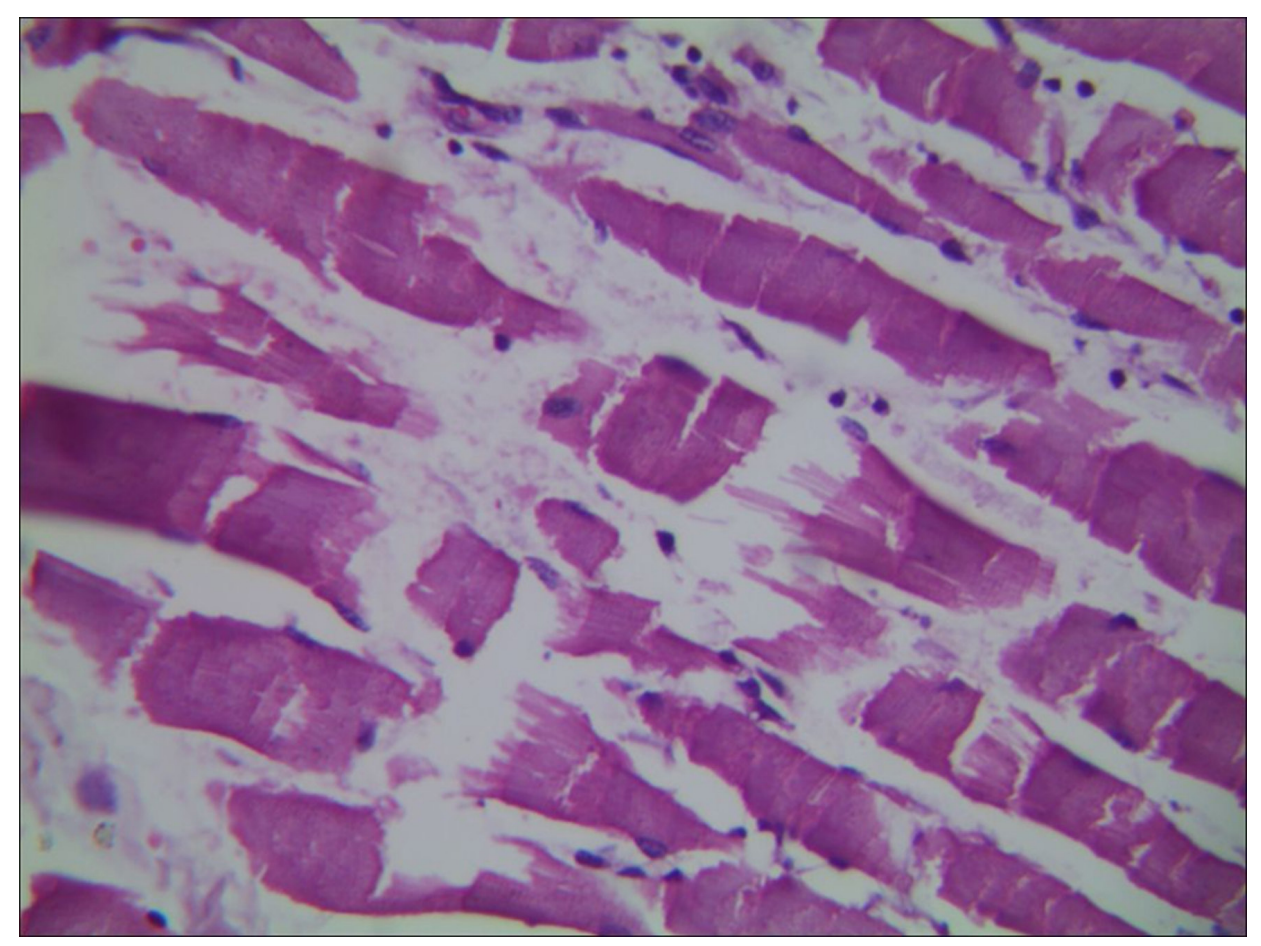

Figure 6. Pathology of the muscle tissues adjacent to the wound tract showed extensive fragmentation. 


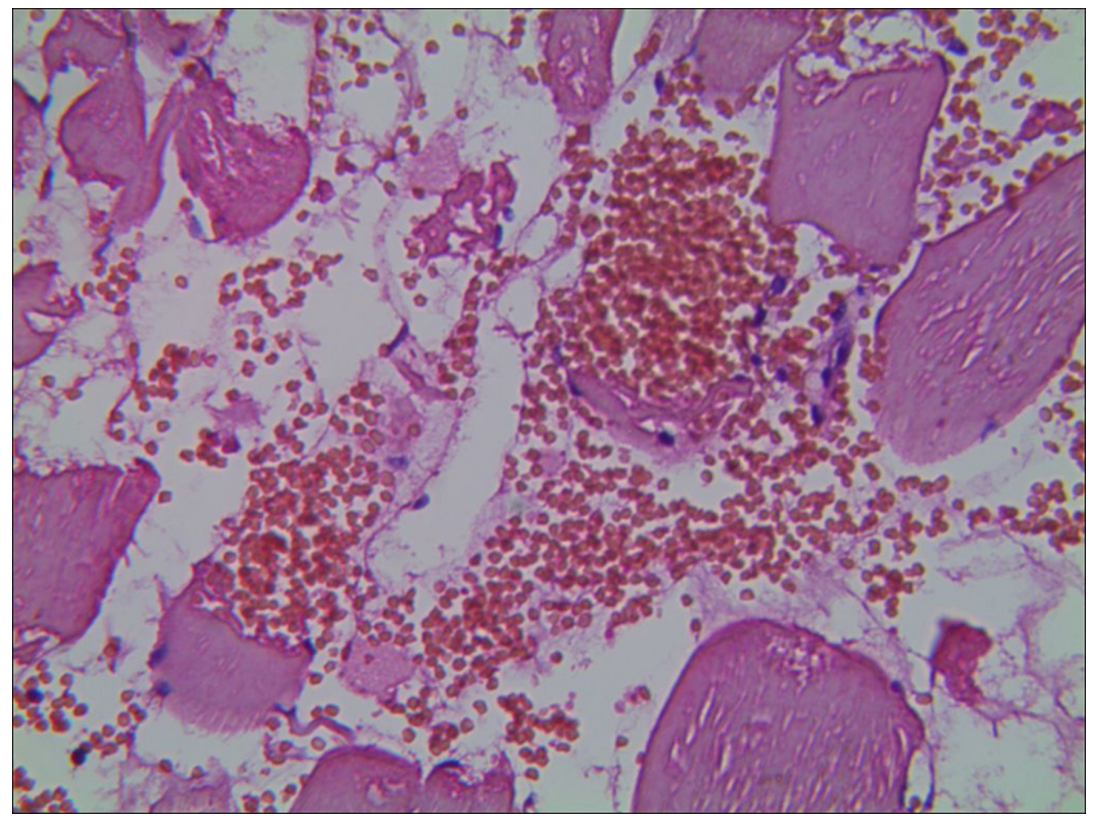

Figure 7. Pathology exhibited the hematocele among the muscle groups.

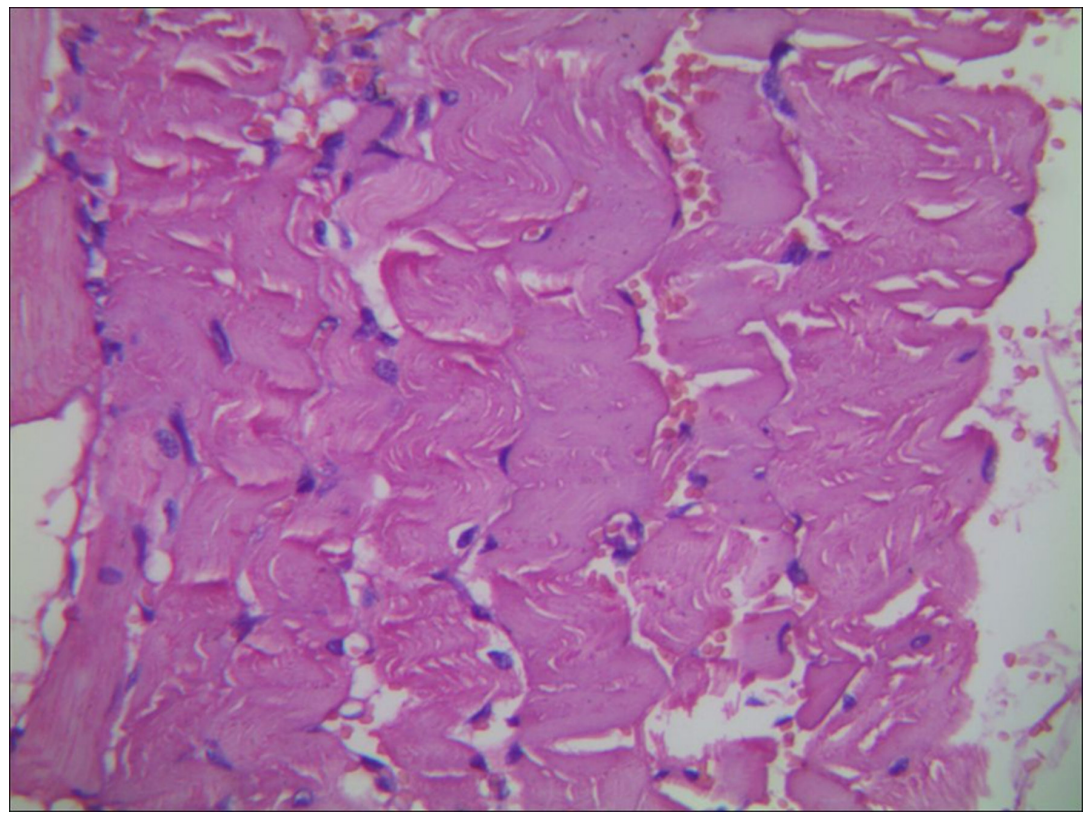

Figure 8. Muscle cells outside wound tract region were cloudy, swelling, and loose with partial wavy changes. 


\section{DISCUSSION}

In the rescue of war wounds, the positioning of the wound tract can depend on personal experience and naked eye observation. The " $4 \mathrm{C}$ " method of intraoperative muscle necrosis judgment, induced by Scully (Olson, 1996), which includes muscle tissue color, blood circulation, muscle contraction, and muscle toughness changes observed with the naked eye, was simple, but imprecise, and was not useful for finding the wound tract. This may lead to tissue injury expansion or even misdiagnosis for treatment, delaying diagnosis. The treatment of gunshot wounds is controversial. Inactivated organs in the wound tract are a good medium for bacteria (Bowyer, 2006) and should be promptly removed. However, many scholars do not advocate complete debridement (Ziv et al., 1989; Bowyer and Rossiter, 1997; MacFarlane, 1999), generally emphasizing the importance of postoperative drainage mainly because the early wound tract in gunshot and the inactivated surrounding tissues are not easy to discern (Fackler et al., 1989). Improper handling may increase defects in trauma-surrounding tissues or damage nearby blood vessels. Stromberg (1978) also reported 41 cases of low-speed gunshot wound; after careful debridement and combination treatment with antibiotics, the patients obtained adequate postoperative drainage and the wound completely closed in stage I without serious complications. Thus, the rapid and accurate identification of the wound tract extension is a prerequisite for minimizing damage towards the normal tissues during debridement as well as the adequate postoperative drainage.

Pathologically, partially damaged tissues can be divided into 3 regions outwards from the wound tract, including the primary wound tract region, the contusion region, and the concussion region (Liu et al., 1991). Klein et al. (1975) suggested that foreign bodies in the wound tract, tissue damage, necrosis, shock, and local circulatory disorders can affect wound repair. Thus, correctly identifying the damaged areas is important for achieving reasonable debridement. Our results showed that over time, the echo in the wound tract gradually increased and became variable; the irregular strong tubular echo could be observed around the tract $(24 \mathrm{~h}$ later), which was the result of blood-clot-constant-activation. In the 2-D ultrasound, although the 3 regions of the traumatic wound tract were not clear, these regions still may be useful for making an objective assessment regarding the injury. Next, we assumed that the ultrasound imaging method could be used to accurately identify the 3 regions of the wound tract based on the 2-D ultrasound, while specific implementation measures were still unclear. Even after the formation of the wound tract over a long period of time $(168 \mathrm{~h})$, the high-resolution ultrasound clearly showed the wound tract extension. Although as time increased, the ultrasound did not show quantitative indicators for the wound tract echo, there was some regularity and the information enabled general judgment regarding injury time, providing an important basis for cleaning of the foreign bodies and necrotic tissues in the wound tract.

The running tract of the high-speed projectile (such as a bullet and fragment) in the body is not as simple as the continuation of the flight tract in the air. The wound path is not straight, particularly in gunshot wounds in the limbs. Because of anatomical specificity, this can easily result in complex damage to the soft tissues, blood vessels, nerves, and bones. In this study, extension of the wound track was slightly tortuous, and the inner diameter was variable, appearing as a small inlet with a large outlet because after the bullet broke into the body tissues, the nutation angle increased significantly, increasing the damage range on the outlet much more than that to the inlet section (Fackler et al., 1988). After the injured limbs were changed from the suspended state to the buckling state, the wound tract immediately became 
discontinuous, with several pipe-shaped echo far away from each other. The corresponding part of the contralateral limb muscles also appeared to show movement. The occurrence of this phenomenon was based on the anatomy and physiological characteristics of the limbs. Limb muscles segmentally distributed based on muscle groups, as the projecting path typically has a certain angle with the body axis, resulting in the wound tract extending into different muscles. With posture changes, the muscles will move along the direction of the force, changing the wound tract extension. Similarly, when recovering to the injured posture, the muscle force returns to its original position, restoring the original continuous state of the wound tract. We observed one relatively simple wound tract among many complex wound tracts (different projectiles, injured sites, or injured posture may result in several disconnected tract segments that are far away from each other). However, when the injury occurred, the injured limb may be in the upright posture or the buckling state, and thus the bullet may penetrate both the thigh and leg, forming a more complex wound tract. Because it may be difficult for the injured person to obtain treatment immediately after injury, when the patient is sent to the hospital, the injured limb should be in the active position, but typically is likely to have been changed position. In this case, if the doctor's experience is the only method for identifying the tract during surgery, it would be difficult to find the debridement, which may increase damage to the normal body tissues and prevent unobstructed drainage. Therefore, before debridement, high-resolution ultrasound can be performed to understand the posture and the wound tract extension when injured, which would facilitate the implementation of debridement and postoperative unobstructed drainage.

High-speed projectiles can cause tissue damages on the trajectory of projectiles; furthermore, the unique "instant cavity effect" can also transfer the energy into the surrounding tissues of the wound tract, bringing air into the wound tract (Fackler, 1996). In this study, high-resolution ultrasound images obtained immediately to visualize wound tract formation were irregular, banded-strong gas echo between the inlet and the outlet, while the echo of the surrounding tissues increased and the texture was disordered, supporting the "instant cavity effect". Over time, the gas in the wound tract reduced, possibly because the limb movements of the laboratory animals during the observation period gradually pressed the residual air outside of the wound tract. We also found that the gas was not only present in the wound tract, but also we incidentally detected a strong gas echo appeared in the surrounding tissues around the wound tract as well as in distant tissues. We injected saline into the wound tract to determine whether these strong gas echoes were related to the wound tract. Surprisingly, after saline injection, not only the wound tract was torn open, but also the adjacent fascia. Thus, these regions are interconnected, allowing for the observed gas migration. We reasoned that this may be because the "instant cavity effect" transferred energy to and damaging the surrounding tissues, while the strong pressure tore the fascia and allowed gas to migrate among the fascia. The characteristic of gas migration to the surrounding tissues provided the evidence of damages of the tissue and fascia surrounding the wound tract.

There were some limitations to our study. First, the sample size was small, although we believe that the results of the small samples are representative. Second, the 53-type musket used in this experiment had no rifling, and thus the projectile had nearly no rotation, so the wound tract was simple. After the posture change, the wound tract broken into segments, producing more complex wound tract areas, but the wound was the simplest type of complex wound tract. The firing rate of $400 \mathrm{~m} / \mathrm{s}$ was much lower than the injuring velocity under war conditions, which is typically $900-1000 \mathrm{~m} / \mathrm{s}$ (Wang, 2000). Therefore, the actual situation is 
more complex and further studies should be conducted to examine this. Finally, this study was conducted using animals and had not been applied in practical clinical applications; thus, our next study will evaluate the clinical effect.

\section{CONCLUSIONS}

In summary, ultrasound not only showed excellent resolution capabilities for examining soft tissues, but is also easy to transport and conducive for on-spot emergencies. Ultrasound imaging of gunshot wounds in pig soft tissues revealed detailed features. High-frequency ultrasound can not only clearly show the wound tract morphology at different time points after injury, but also accurately identify complex wound tract extensions because of postural changes. The specific characteristic of gas migration along the fascia to the wound tract-surrounding tissues revealed by ultrasound provided evidence of damage to the tissues and fascia surrounding the wound tract. Ultrasound technology applied for gunshot wounds can provide unobstructed imaging and facilitate clinical treatment, particularly in the implementation of debridement and postoperative unobstructed drainage, and help improve the judgment and treatment of limb gunshot injuries.

\section{REFERENCES}

Bowyer G (2006). Debridement of extremity war wounds. J. Am. Acad. Orthop. Surg. 14: S52-S56.

Bowyer GW and Rossiter ND (1997). Management of gunshot wounds of the limbs. J. Bone Joint Surg. Br. 7926: 10311036.

Coupland R (1994). War wounds of bones and external fixation. Injury 25: 211-217.

Fackler ML (1996). Gunshot wound review. Ann. Emerg. Med. 28: 194-203.

Fackler ML, Bellamy RF and Malinowski JA (1988). The wound profile: illustration of the missile-tissue interaction. $J$. Trauma 28: S21-S29.

Fackler ML, Breteau J, Courbil L, Taxit R, et al. (1989). Open wound drainage versus wound excision in treating the modern assault rifle wound. Surgery 105: 576-584.

Kim PE, Go JL and Zee CS (2002). Radiographic assessment of cranial gunshot wounds. Neuroimaging Clin. N. Am. 12: 229-248.

Klein RS, Berger SA and Yekutiel P (1975). Wound infection during the Yom Kippur war: observations concerning antibiotic prophylaxis and therapy. Ann. Surg. 182: 15-21.

Liu YQ, Wang ZG and Ma YY (1991). Wound allistics. People's Military Medical Publishing House, Beijing, pp. 174195.

MacFarlane C (1999). Management of gunshot wounds: the Johannesburg experience. Int. Surg. 84: 93-98.

Olson SA (1996). Open fractures of the tibial shaft. J. Bone Joint Surg. 78: 1428-1437.

Parker S, Jarvis L and Dale R (1997). Magnetic resonance imaging in the evaluation of a high-velocity gunshot wound to the thigh. Br. J. Surg. 84: 1119.

Sadjadi J, Cureton EL, Dozier KC, Kwan RO, et al. (2009). Expedited treatment of lower extremity gunshot wounds. $J$. Am. Coll. Surg. 209: 740-745.

Schwartz RB (1995). Helical (spiral) CT in neuroradiologic diagnosis. Radiol. Clin. North Am. 33: 981-995.

Stanec Z, Skrbić S, Depina I, Hulina D, et al. (1993). Surgical management of war injuries involving soft tissue defects. Lijec. Vjesn. 115: 283-289.

Stromberg BV (1978). Management of low-velocity gunshot wounds of the hand. South Med. J. 71: 1087-1088.

Tartaglione T, Filograna L, Roiati S, Guglielmi G, et al. (2012). Importance of 3D-CT imaging in single-bullet cranioencephalic gunshot wounds. Radiol. Med. 117: 461-470.

Tayal VS, Beatty MA, Marx JA, Tomaszewski CA, et al. (2004). FAST (focused assessment with sonography in trauma) accurate for cardiac and intraperitoneal injury in penetrating anterior chest trauma. J. Ultrasound Med. 23: 467-472.

Truax AL, Chandnani VP, Chacko AK and Gonzalez DM (1997). Incidence and methods of diagnosis of musculoskeletal injuries incurred in Operations Desert Shield and Desert Storm. Invest. Radiol. 32: 169-173. 
Wang ZG (2000). Thinking on wound ballistics research. Chin. J. Traumatol 16: 133-135.

Ziv I, London E, Wishnie P, Scherer P, et al. (1989). One-stage treatment of gunshot wounds. J. Foot Surg. 28: 255258. 